\title{
On nonlinear dynamics and control of a robotic arm with chaos
}

\author{
J. L. P. Felix ${ }^{1}$, E. L. Silva ${ }^{1}$, J. M. Balthazar ${ }^{2}$, A. M. Tusset ${ }^{3}$, A. M. Bueno ${ }^{4}$, R. M. L. R. F. Brasil ${ }^{2}$ \\ ${ }^{1}$ UNIPAMPA, Bagé, RS, Brazil \\ ${ }^{2}$ UFABC, Santo Andre, SP, Brazil \\ ${ }^{3}$ UTFPR, Ponta Grossa, PR, Brazil \\ ${ }^{4}$ UNESP, Sorocaba, SP, Brazil
}

\begin{abstract}
In this paper a robotic arm is modelled by a double pendulum excited in its base by a DC motor of limited power via crank mechanism and elastic connector. In the mathematical model, a chaotic motion was identified, for a wide range of parameters. Controlling of the chaotic behaviour of the system, were implemented using, two control techniques, the nonlinear saturation control (NSC) and the optimal linear feedback control (OLFC). The actuator and sensor of the device are allowed in the pivot and joints of the double pendulum. The nonlinear saturation control (NSC) is based in the order second differential equations and its action in the pivot/joint of the robotic arm is through of quadratic nonlinearities feedback signals. The optimal linear feedback control (OLFC) involves the application of two control signals, a nonlinear feedforward control to maintain the controlled system to a desired periodic orbit, and control a feedback control to bring the trajectory of the system to the desired orbit. Simulation results, including of uncertainties show the feasibility of the both methods, for chaos control of the considered system.
\end{abstract}

\section{Introduction}

Dynamically systems with pendulums have important applications. An auto parametric non-ideal system with pendulum was studied by [1], and an auto parametric system with two pendulums harmonically excited was studied by [2].

The first detailed study on non-ideal vibrating systems was done by [3]. After that publication, the problem of non-ideal vibrating systems has been investigated by a number of authors. A complete review of different theories on non-ideal vibrating systems is to be found in [4]. Dynamic interactions between a parametric pendulum and an electro-dynamical shaker of limited power was investigated by [5]. In That paper a mathematical model of the electromechanical shaker was described and its parameters were identified.

The effectiveness of the nonlinear saturation control in vibration attenuation for a non-ideal portal frame was investigated by [6].

The Optimal Linear Feedback Control was proposed by [7]. In [7] the quadratic nonlinear Lyapunov function was proposed to solve the optimal nonlinear control design problem for a nonlinear system. [8] formulated the linear feedback control strategies for nonlinear systems, asymptotic stability of the closed-loop nonlinear system, guaranteeing both stability and optimality.
We organized this paper as follows. In section 2 we obtain the mathematical model and perform the analysis of the dynamic model considering: bifurcation diagrams, time histories, phase portraits, frequency spectrum, and 01 test for chaotic behaviour. In section 3 , the nonlinear saturation control (NSC) and the optimal linear feedback control (OLFC) are implemented. In section 4, the efficiency and the robustness to parametric errors of each control technique are verified through computer simulations. Finally, some concluding remarks are given.

\section{System description and governing equations}

We consider a robotic arm modelled by a double pendulum excited in its base by a DC motor of limited power via a crank mechanism and a spring, displayed in Fig. 1. The supporting elastic substructure of the robotic arm consist of a mass, spring and damper $(m, k, c)$ whose motion is in the vertical direction. The length and mass of the two parts of the robotic arm are $l_{1}, l_{2}, m_{1}$, $m_{2}$, whose angular deflections are measured from the vertical line $\left(\theta_{1}, \theta_{2}\right)$. The controlled torque of the unbalanced DC motor is considered as a linear function of its angular velocity, $\Gamma(\dot{\varphi})=V_{m}-C_{m} \dot{\varphi} . V_{m}$ is set as our 
control parameter and it changes according to the applied voltage, $C_{m}$ is a constant for each model of motor considered. The coupling between the DC motor and robotic arm is acomplished by a crank mechanism of radius $r$ and elastic connector of stiffness $k_{r}$.

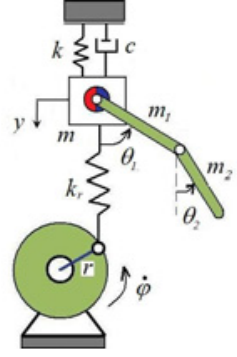

Fig. 1. Robotic arm excited by a non-ideal motor via crankspring mechanism.

From the Lagrange's method, the equations of motion for the system are:

$$
\begin{aligned}
& \left(m+m_{1}+m_{2}\right) \ddot{y}+c \dot{y}+k y-l_{1}\left(m_{1}+m_{2}\right) \ddot{\theta}_{1} \sin \theta_{1} \\
& -l_{2} m_{2} \ddot{\theta}_{2} \sin \theta_{2}-\left(m_{1}+m_{2}\right) l_{1} \dot{\theta}_{1}^{2} \cos \theta_{1} \\
& -m_{2} l_{2} \dot{\theta}_{2}^{2} \cos \theta_{2}=k_{r} r \sin \varphi \\
& I \ddot{\varphi}=\Gamma(\dot{\varphi})+k_{r} r(y-r \sin \varphi) \cos \varphi \\
& \left(m_{1}+m_{2}\right) l_{1}^{2} \ddot{\theta}_{1}+m_{2} l_{1} l_{2} \ddot{\theta}_{2} \cos \left(\theta_{1}-\theta_{2}\right) \\
& -m_{2} l_{1} l_{2} \dot{\theta}_{2}^{2} \sin \left(\theta_{2}-\theta_{1}\right)+\left(m_{1}+m_{2}\right) g l_{1} \sin \theta_{1} \\
& +c_{1} \dot{\theta}_{1}-c_{2}\left(\dot{\theta}_{2}-\dot{\theta}_{1}\right)=\left(m_{1}+m_{2}\right) l_{1} \ddot{y}_{1} \sin \theta_{1} \\
& m_{2} l_{2}^{2} \ddot{\theta}_{2}+m_{2} l_{1} l_{2} \ddot{\theta}_{1} \cos \left(\theta_{2}-\theta_{1}\right)+m_{2} g l_{2} \sin \theta_{2} \\
& +m_{2} l_{1} l_{2} \dot{\theta}_{1}^{2} \sin \left(\theta_{2}-\theta_{1}\right)+c_{2}\left(\dot{\theta}_{2}-\dot{\theta}_{1}\right)=m_{2} l_{2} \ddot{y} \sin \theta_{2}
\end{aligned}
$$

Next, we use the following changes of variables:

$\tau=\omega_{g} t, \quad y_{0}=\frac{y}{l_{1}}, \quad \omega_{0}^{2}=\frac{k}{m_{t}}, \quad m_{t}=m+m_{1}+m_{2}$,

$\hat{\Gamma}(\dot{\varphi})=a-b \dot{\varphi}, \quad \omega_{1}^{2}=\frac{g}{l_{1}}, \quad \omega_{2}^{2}=\frac{g}{l_{2}}, \quad \alpha_{1}=\frac{m_{1}+m_{2}}{m_{t}}$, $\alpha_{2}=\frac{m_{2}}{m_{t}}, R=\frac{l_{2}}{l_{1}}, \eta_{1}=\frac{k_{r} r}{k l_{1}}, \alpha_{3}=\frac{m_{2}}{m_{1}+m_{2}}, \Omega_{1}=\frac{\omega_{1}}{\omega_{0}}$, $\Omega_{2}=\frac{\omega_{2}}{\omega_{0}}, \quad \mu_{1}=\frac{c_{1}}{\left(m_{1}+m_{2}\right) \omega_{0} l_{1}^{2}}, \quad \mu_{2}=\frac{c_{2}}{\left(m_{1}+m_{2}\right) \omega_{0} l_{1}^{2}}$, $\mu_{3}=\frac{c_{2}}{m_{2} \omega_{0} l_{2}^{2}}, \eta_{2}=\frac{k_{r} r l_{1}}{I \omega_{0}^{2}}, \eta_{3}=\frac{k_{r} r^{2}}{I \omega_{0}^{2}}$.

to render the equations dimensionless, in state variables:

$$
\left\{\begin{array}{l}
x_{1}^{\prime}=x_{2} \\
x_{2}^{\prime}=\alpha_{1} x_{4}^{\prime} \sin x_{3}+R \alpha_{2} \sin x_{5}+\alpha_{1} x_{4}^{2} \cos x_{3} \\
+R \alpha_{2} x_{6}^{2} \cos x_{5}-\mu_{0} x_{2}-x_{1}+\eta_{1} \sin x_{7}
\end{array}\right.
$$

$$
\left\{\begin{array}{l}
x_{3}^{\prime}=x_{4} \\
x_{4}^{\prime}=\alpha_{3} R x_{6}^{\prime} \cos \left(x_{5}-x_{3}\right)+x_{2}^{\prime} \sin x_{3}-\Omega_{1}^{2} \sin x_{3} \\
+R \alpha_{3} x_{6}^{2} \sin \left(x_{5}-x_{3}\right)-\mu_{1} x_{4}+\mu_{2}\left(x_{6}-x_{4}\right)
\end{array}\right.
$$

$$
\begin{aligned}
& \left\{\begin{array}{l}
x_{5}^{\prime}=x_{6} \\
x_{6}^{\prime}=-\frac{1}{R} x_{4}^{\prime} \cos \left(x_{5}-x_{3}\right)+\frac{1}{R} x_{2}^{\prime} \sin x_{5}-\Omega_{2}^{2} \sin x_{5} \\
-\frac{1}{R} x_{4}^{2} \sin \left(x_{5}-x_{3}\right)-\mu_{3}\left(x_{6}-x_{4}\right)
\end{array}\right. \\
& \left\{\begin{array}{l}
x_{7}^{\prime}=x_{8} \\
x_{8}^{\prime}=a-b x_{8}+\left(\eta_{2} x_{1}-\eta_{3} \sin x_{7}\right) \cos x_{7}
\end{array}\right.
\end{aligned}
$$

The adopted state variables are: $x_{1}=y_{0}, x_{2}=\dot{y}_{0}, x_{3}=\theta_{1}$, $x_{4}=\dot{\theta}_{1}, \quad x_{5}=\theta_{2}, \quad x_{6}=\dot{\theta}_{2}, \quad x_{7}=\varphi, \quad x_{8}=\dot{\varphi}$. For the numerical simulations, we used the following dimensionless parameters: $\alpha_{1}=0.3 ; \alpha_{2}=0.17 ; \alpha_{1}=0.5$

; $R=1 ; \quad \mu_{1}=0.01 ; \quad \mu_{2}=0.01 ; \mu_{3}=0.01 ; a=1.22$; $b=1.2 ; \Omega_{1}=0.9 ; \quad \Omega_{2}=0.4 ; \quad \eta_{1}=0.05 ; \quad \eta_{2}=0.2$; $\eta_{2}=0.3$. We used the following initial conditions: $\left[\begin{array}{llllllll}0 & 0 & 0 & 0 & \frac{5 \pi}{180} & 0 & 0 & 0\end{array}\right]$.

Figure 2 shows the bifurcation diagram for parameter $\mu_{0}$.

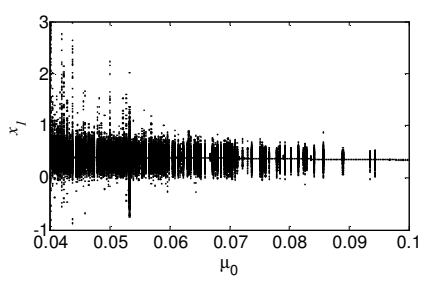

Fig. 2. Bifurcation diagram for $\mu_{0}$.

In Fig. 2, we can see that for some values of parameter $\mu_{0}$ the system (9-11) has chaotic behaviour. To determine these values of parameter $\mu_{0}$ we applied the 0 1 test to verify chaotic behaviour of the system, as detailed in [9]. The 0-1 test for chaos takes as input a time series of measurements and returns a single scalar value of either 0 for periodic attractors or 1 for chaotic attractors [9].

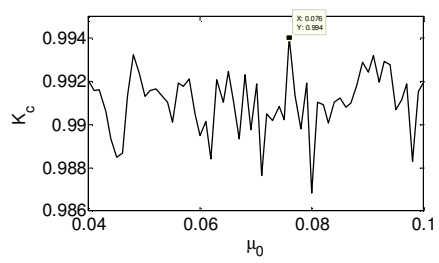

Fig. 3. Asymptotic growth rate $\left(K_{c}\right)$ from $0-1$ test as a function of parameter $\mu_{0}$. 
According to [9] the value of $K_{c}$ is given by:

$$
K_{c}=\frac{\operatorname{cov}(X, M(c))}{\sqrt{\operatorname{var}(X) \operatorname{var}(M(c))}}
$$

where vectors $\mathrm{X}=\left[1,2, \ldots, \mathrm{n}_{\max }\right]$, and $\mathrm{M}(\mathrm{c})=[\mathrm{M}(1, \mathrm{c}), \ldots$, $\left.\mathrm{M}\left(1, \mathrm{n}_{\max }\right)\right], c \in(0, \pi)$ is a fixed frequency arbitrarily chosen, and :

$$
\begin{aligned}
& M(n, c)=\lim _{N \rightarrow \infty} \frac{1}{N} \sum_{j=1}^{N}\left[\begin{array}{l}
(p(j+n)-p(j))^{2}+ \\
+(q(j+n)-q(j))^{2}
\end{array}\right] \\
& p(i)=\sum_{j=0}^{i} \frac{\left(x_{j}-\bar{x}\right)}{\sigma_{x}} \cos (j c) ; \quad q(i)=\sum_{j=0}^{i} \frac{\left(x_{j}-\bar{x}\right)}{\sigma_{x}} \sin (j c)
\end{aligned}
$$

where $\bar{x}$ and $\sigma_{x}$ are the mean value and square deviation of the examined $x_{i}$ series, and $N$ is the length of the sampled points in the displacement time series.

A value of $K_{c} \cong 0$ indicates a non-chaotic data set while a value of $K_{c} \cong 1$ indicates a chaotic data set. As we can see in Fig. 3, for $\mu_{0}=0.076$ system (5)-(8) has chaotic behaviour.

In Figs. (4-6) one can observe the behaviour of the system (5)-(8) for $\mu_{0}=0.076$.

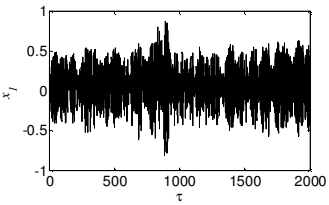

(a)

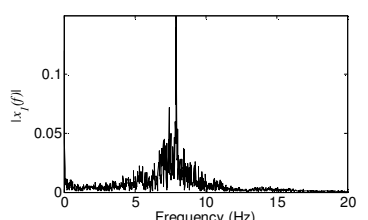

(b)
Fig. 4. Behaviour $x_{1}$. (a) Vertical movement. (b) Frequency Spectrum

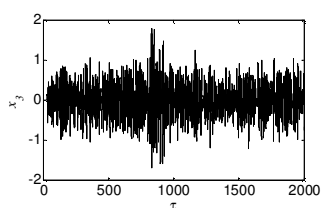

(a)

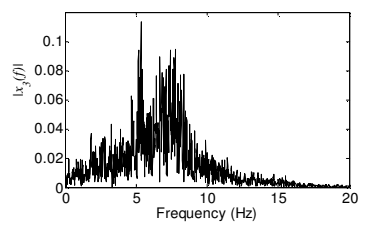

(b)
Fig. 5. Behaviour $x_{3}$. (a) Angular movement. (b) Frequency Spectrum

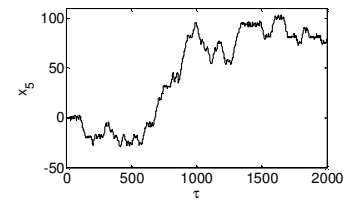

(a)

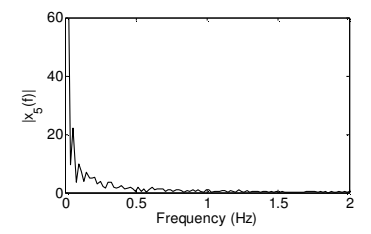

(b)
Fig. 6. Behaviour $x_{5}$. (a) Angular movement. (b) Frequency Spectrum
Figures (4-6) show the chaotic behaviour of the system (5)-(8).

\section{PROPOSED CONTROL}

With the objective of eliminate the chaotic behaviour of the system (9)-(12), we considered the introduction of a control signal $U$ to the system (9), as shown in Fig. 7Ç

$\left\{\begin{array}{l}x_{1}^{\prime}=x_{2} \\ x_{2}^{\prime}=\alpha_{1} x_{4}^{\prime} \sin x_{3}+R \alpha_{2} \sin x_{5}+\alpha_{1} x_{4}^{2} \cos x_{3} \\ +R \alpha_{2} x_{6}^{2} \cos x_{5}-\mu_{0} x_{2}-x_{1}+\eta_{1} \sin x_{7}+U\end{array}\right.$

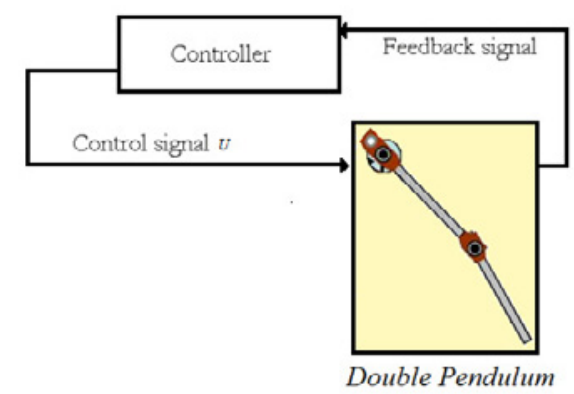

Fig. 7. Control applied in pivot and joint of the robotic arm asymptotic growth rate

\subsection{Formulation of nonlinear saturation control (NSC)}

In this section, we implement the nonlinear saturation control:

$U=\gamma_{1} u^{2}$

$u$ is obtained from the following equation:

$\ddot{u}+\mu_{c} \dot{u}+\omega_{c}^{2} u=\gamma_{2} y_{0} u$

where $\omega_{c}$ is the controller's natural frequency, $\gamma_{1}$ and $\gamma_{2}$ are positive constants. Internal resonance conditions are considered by letting $2 \omega_{c} \approx 1$ and external resonance by $\dot{\varphi} \approx 1$.

In Fig. 8 we can observe the behaviour of the system (5)(8) with the proposed control (13) considering the following parameters: $\quad \gamma_{1}=0.01 ; \quad \gamma_{2}=0.07$; $\mu_{c}=0.01 ; \omega_{c}=0.01$ and initial conditions: $u(0)=0.1$ and $\dot{u}(0)=0$, excluding the transient behavior. 


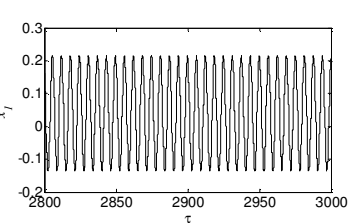

(a)

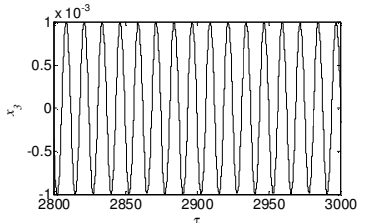

(b)

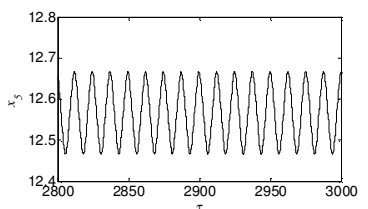

(c)

Fig. 8. (a) Vertical motion $x_{1}$. (b) Angular motion $x_{3}$. (c) Angular motion $x_{5}$

\subsection{Control using optimal linear feedback control (OLFC)}

Next, we present the optimal linear control strategy for nonlinear systems [10]. It is important to observe that this approach is analytical, without dropping any nonlinear term [11].

The $U$ vector control in equation (12) consists of two parts: $U=u_{f f}+u_{f b}$, where $u_{f f}$ is the feed-forward control and $u_{f b}$ is the linear feedback control. We define the period orbit as a function of $\left[x_{1}^{*}(\tau) \quad x_{2}^{*}(\tau)\right]^{T}$. If the function $\left[x_{1}^{*}(\tau) \quad x_{2}^{*}(\tau)\right]^{T}$ is the solution of (12), without the control $U$, then $u_{f b}=0$.

In this way, the desired regime is obtained by the following equation:

$$
\left\{\begin{array}{l}
x_{1}^{*}=x_{2}^{*} \\
x_{2}^{*}=\alpha_{1} x_{4}^{\prime} \sin x_{3}+R \alpha_{2} \sin x_{5}+\alpha_{1} x_{4}^{2} \cos x_{3} \\
+R \alpha_{2} x_{6}^{2} \cos x_{5}-\mu_{0} x_{2}^{*}-x_{1}^{*}+\eta_{1} \sin x_{7}+u_{f f}
\end{array}\right.
$$

The feed-forward control $u_{f f}$ is given by:

$u_{f f}=x_{2}^{*}-\alpha_{1} x_{4}^{\prime} \sin x_{3}-R \alpha_{2} \sin x_{5}-\alpha_{1} x_{4}^{2} \cos x_{3}$

$-R \alpha_{2} x_{6}^{2} \cos x_{5}+\mu_{0} x_{2}^{*}+x_{1}^{*}-\eta_{1} \sin x_{7}$

Substituting (16) into (12) and defining the deviation of the desired trajectory as:

$z=\left[\begin{array}{l}x_{1}-x_{1}^{*} \\ x_{2}-x_{2}^{*}\end{array}\right]$

the system can be represented, in matrix form $z^{\prime}=A z+B u$ : $\left[\begin{array}{l}z_{1}^{\prime} \\ z_{2}^{\prime}\end{array}\right]=\left[\begin{array}{cc}0 & 1 \\ -1 & -\mu_{0}\end{array}\right]\left[\begin{array}{l}z_{1} \\ z_{2}\end{array}\right]+\left[\begin{array}{l}0 \\ 1\end{array}\right] u_{f b}$

Control $u_{f b}$ can be found solving the following equation:

$u_{f b}=-R^{-1} B^{T} P z$

$P$ is a symmetric matrix symmetrically and may be found solving the Algebraic Riccati Equation:

$P A+A^{T} P-P B R^{-1} B^{T} P+Q=0$

where: $Q$ symmetrical, positive definite, and $R$ positive definite, ensuring that the control (19) is optimal [12].

We define the desired periodic orbits obtained with the nonlinear saturation control Fig. 8a through the use of Fourier series, calculated numerically as:

$\left\{\begin{array}{l}x_{1}^{*}=0.0393+0.1749 \sin \left(\frac{2 \pi}{7} \tau\right) \\ x_{2}^{*}=\frac{0.3498 \pi}{7} \cos \left(\frac{2 \pi}{7} \tau\right)\end{array}\right.$

Matrices $A$ and $B$ are:

$A=\left[\begin{array}{cc}0 & 1 \\ -1 & -0.076\end{array}\right], B=\left[\begin{array}{l}0 \\ 1\end{array}\right]$

Defining:

$Q=\left[\begin{array}{rc}10^{4} & 0 \\ 0 & 10\end{array}\right], R=[10]$

and solving the Algebraic Riccati Equation (20), we obtain:

$P=\left[\begin{array}{cc}1442.2713 & 99.005 \\ 99.005 & 14.3467\end{array}\right]$

Substituting it into (20), we define the control:

$u_{f b}=-99.005 z_{1}-14.3467 z_{2}=$

$-99.005\left(x_{1}-x_{1}^{*}\right)-14.3467\left(x_{2}-x_{2}^{*}\right)$

Considering (25) and (16) we obtain $U$ :

$U=-99.005\left(x_{1}-x_{1}^{*}\right)-14.3467\left(x_{2}-x_{2}^{*}\right)$

$+x_{2}^{*}-\alpha_{1} x_{4}^{\prime} \sin x_{3}-R \alpha_{2} \sin x_{5}-\alpha_{1} x_{4}^{2} \cos x_{3}$

$-R \alpha_{2} x_{6}^{2} \cos x_{5}+\mu_{0} x_{2}^{*}+x_{1}^{*}-\eta_{1} \sin x_{7}$

In Fig. 9, one observes the controlled system (5)-(8) in the orbit (21), with: $\left|x_{1}-x_{1}^{*}\right|<10^{6}$, excluding the transient behavior. 


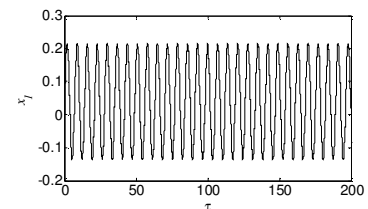

(a)

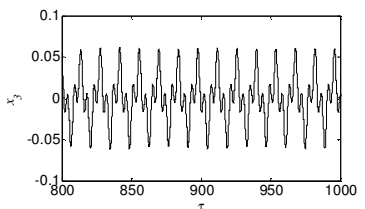

(b)

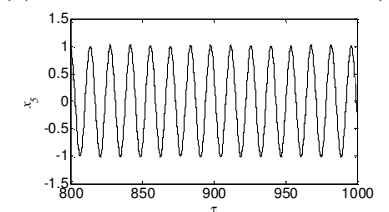

(c)

Fig. 9. (a) Vertical motion $x_{1}$. (b) Angular motion $x_{3}$. (c)

$$
\text { Angular motion } x_{5}
$$

As it can be seen, the proposed control (26), took the system to the desired orbit (21), with transient less than $2 \tau$.

\subsection{Comparison between NSC control and OLFC Control}

In Fig. 10 we can observe the behavior of the system (5)(8) using NSC control and OLFC control.

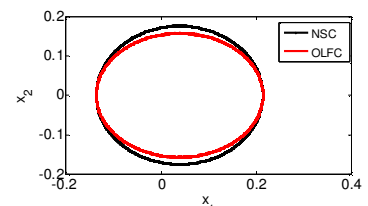

(a)

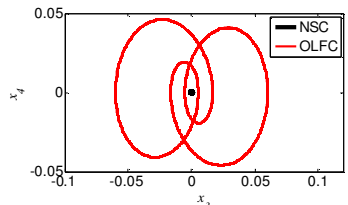

(b)

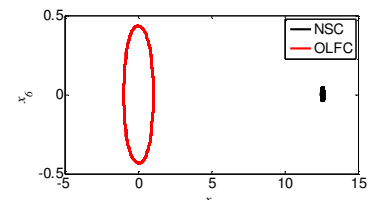

(c)

Fig. 10. Phase diagram. (a) Motion $x_{1}$. (b) Angular motion $x_{3}$

(c) Angular motion $x_{5}$

In Fig. 11 we can see the variation of the control signal used to control NSC and OLFC control.

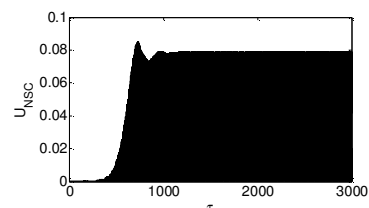

(a)

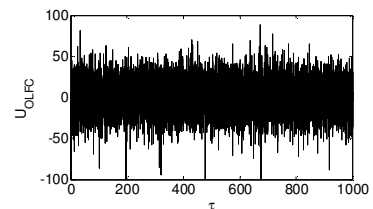

(b)
Fig. 11. Control signal U. (a) signal used in NSC control. (b) signal used in OLFC

As can be seen in Fig. 11, to eliminate the transient and maintain the system in a defined orbit, the OLFC control used a signal more intense than that used by NSC control. We can also observe in Fig. 10 that even with $x_{1}$ being similar for both controls we do not get the same behavior for other states.

\section{Control in the presence of parametric errors}

To consider the effect of parameter uncertainties on the performance of the controller, the parameters used in the control will be considered having a random error of $\pm 20 \%$ [13-14]. A sensitivity analysis will be carried out considering the error: $e_{i}=x_{i}-\tilde{x}_{i}$ for $i=1: 6 . x_{i}$ is the state of the system with control without parametric error, and $\tilde{x}_{i}$ for the control with parametric error.

\subsection{Nonlinear saturation control (NSC) with parametric error}

In Fig. 11 we can observe the periodic behavior for the system (5)-(8) with the following control (13): $\gamma_{1}=0.008+0.004 r(t) ; \quad \gamma_{2}=0.056+0.028(t)$; $\mu_{c}=0.008+0.004 r(t) ; \quad \omega_{c}=0.008+0.004 r(t)$, excluding the transient behavior. $r(t)$ is a normally distributed random function.

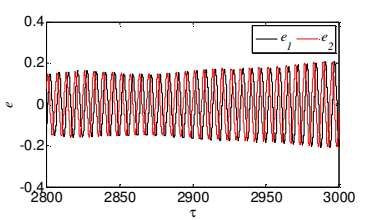

(a)

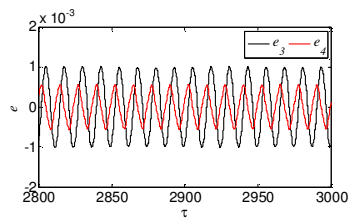

(b)

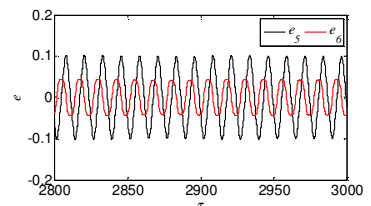

(c)

Fig. 11. Sensitivity of NSC control to parametric errors

In Fig. 12 we can observe the periodic behavior for the system (5)-(8) with the following control (26): $\alpha_{1}=0.24+0.12 r(t) ; \quad \alpha_{2}=0.136+0.068(t)$;

$R=0.8+0.4 r(t) ; \quad \quad \mu_{0}=0.0608+0.0304(t)$;

$\eta_{1}=0.04+0.02 r(t)$.

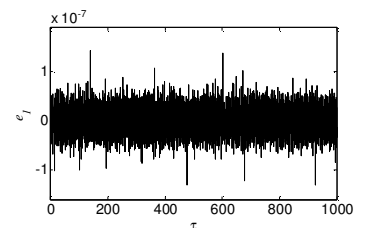

(a)

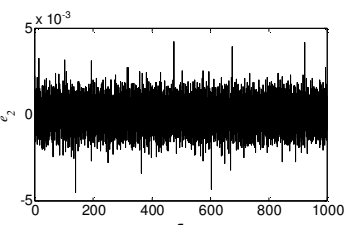

(b) 


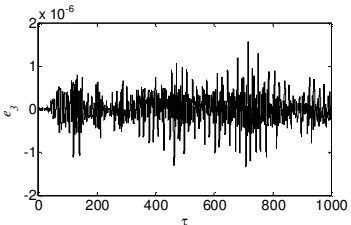

(c)

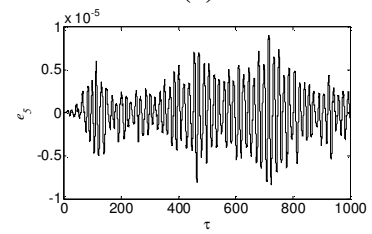

(e)

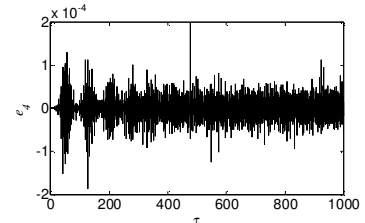

(d)

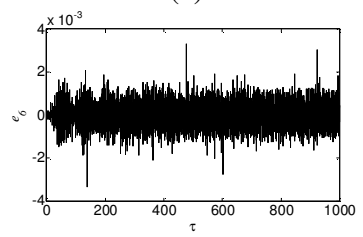

(f)
Fig. 12. Sensitivity of OLFC control to parametric errors

We can see from Fig. 11 that the NSC control is sensitive to parameter uncertainties taking the system to different periodic orbits from those obtained without parameter uncertainties. As it can be seen in Fig. 12, the OLFC control has proven to be robust to parameter uncertainties.

\section{Conclusions}

We consider a robotic arm modeled by a double pendulum excited in its base by a DC motor of limited power via a crank mechanism and a spring. An investigation of the nonlinear dynamics and chaos was carried out based on this model. The results obtained show chaotic behavior of the model and define the parameters for which chaos occurs.

Two control strategies have shown to be effective in stabilizing the system in a periodic orbit. Associating the time delay control to get the desired orbit and the optimal control to maintain the desired orbit we got less transient time and more robustness.

\section{References}

1. D. Sado and M. Kot, Nonlinear oscillations of a coupled autoparametrical system with ideal and nonideal sources of power Mathematical Problems in Engineering, 1-20, (2006)

2. D. Sado and K. Gajos, Note on chaos in three degree of freedom dynamical system with double pendulum, Meccanica, 38, 719-729, (2003).

3. V. O. Kononenko, Vibrating Systems with Limited Power Supply, Illife Books, London, (1969).

4. J. M. Balthazar, D. T. Mook, H. I. Weber, R. M. L. R. F. Brasil, A. Fenili, D. Belato, and J. L. P. Felix, An overview on non-ideal vibrations, Meccanica 38 (6), 613-621, (2003).

5. X. Xu, E. Pavlosvskaia, M. Wiercigroch, F. Romeo and S. Lenci, Dynamic Interactions between Parametric Pendulum and electro-dynamical shaker, ZAMM, 87 (2), 172-186, (2007).

6. J. L. P. Felix, J. M. Balthazar, R. M. L. R. F. Brasil, On saturation control of a non-ideal vibrating portal frame foundation type shear-building, J. Vib. Control 11, 121-136 (2005)
7. M. Rafikov and J. M. Balthazar, On an optimal control design for Rössler system. Phys. Lett. A, 333, 241-245 (2004).

8. M. Rafikov, J. M. Balthazar and A. M Tusset, An Optimal Linear Control Design for Nonlinear Systems. J. of the Braz. Soc. of Mech. Sci. \& Eng., XXX (4), 279-284 (2008).

9. R. Gopal, A. Venkatesan and M. Lakshmanan, Applicability of 0-1 test for strange nonchaotic attractors, Chaos 23, 023123-1:15, (2013).

10. A. M. Tusset, J. M. Balthazar, F. R Chavarette and J. L. P. Felix, On energy transfer phenomena, in a nonlinear ideal and nonideal essential vibrating systems, coupled to a (MR) magneto-rheological damper. Nonlinear Dynamics, 69, 1859-1880, (2012).

11. R. Nozaki, J. M. Balthazar, A. M. Tusset; B. R. Pontes JR and A. M. Bueno,. Nonlinear Control System Applied to Atomic Force Microscope Including Parametric Errors. Journal of Control, Automation and Electrical Systems, 24, p. 223-231, 2013.

12. Pedroso, M. D. ; Nascimento, C. B. ; Tusset, C. M. ; Kaster, M. S., A hyperbolic tangent adaptive pid+lqr control applied to a step-down converter using poles placement design implemented in fpga, Mathematical problems in engineering (print), 2013

13. M. J. Shirazi, R. Vatankhah, M. Boroushaki, H. Salarieh and A. Alasty, Application of particle swarm optimization in chaos synchronization in noisy environment in presence of unknown parameter uncertainty. Communications in Nonlinear Science and Numerical Simulation, 17(2), 742-753 (2012).

14. A. M. Tusset, A. M. Bueno, C. B. Nascimento, M. S. Kaster, J. M. Balthazar, Nonlinear state estimation and control for chaos suppression in MEMS resonator. Shock and Vibration, 20, 749-761, (2013). 